\title{
Efficacy of vertebral augmentation in the treatment of osteoporotic vertebral compression fractures: a systematic review and meta-analysis
}

\author{
Shu Lin ${ }^{1 \#}$, Ming Cheng ${ }^{2 \#}$, Weimin Liang ${ }^{1}$, Xinwei Yuan ${ }^{1}$ \\ ${ }^{1}$ Department of Orthopedics, Sichuan Provincial People's Hospital, University of Electronic Science and Technology of China, Chengdu, China; \\ ${ }^{2}$ Department of Rehabilitation, Jinniu District People's Hospital of Chengdu, Chengdu, China \\ Contributions: (I) Conception and design: S Lin; (II) Administrative support: W Liang, X Yuan; (III) Provision of study materials or patients: M \\ Cheng; (IV) Collection and assembly of data: All authors; (V) Data analysis and interpretation: S Lin; (VI) Manuscript writing: All authors; (VII) \\ Final approval of manuscript: All authors. \\ \#These authors contributed equally to this work. \\ Correspondence to: Weimin Liang; Xinwei Yuan. Department of Orthopedics, Sichuan Provincial People's Hospital, University of Electronic Science and \\ Technology of China, No. 32 West Second Section, First Ring Road, Chengdu 610072, China. Email: liangbsb@126.com; yuanxinwei19870124@163.com.
}

Background: Percutaneous vertebroplasty (PVP) and percutaneous kyphoplasty (PKP) are common
vertebral augmentation (VA) procedures for the treatment of osteoporotic vertebral compression fractures
(OVCF), each with their own advantages and disadvantages. In recent years, the development of new
implant-assisted technologies has provided a breakthrough in VA. This study systematically evaluated and
meta-analyzed the reports on new implant-assisted VA techniques in recent years, so as to provide evidence
for clinical diagnosis and treatment.
Methods: The PubMed, Embase, Ovid, and SpringerLink databases were searched for randomized
controlled studies on VA in the treatment of OVCF. In this study, patients in the experimental group were
treated with PVP using the new implant-assisted VA technique, while patients in the control group were
treated with PKP. Bias assessment was conducted using the tool integrated with the Revman 5.4 software,
and meta-analysis was carried out to compare the mid-term postoperative pain relief, functional status,
quality of life, and cement extravasation between the two groups (each presented with a forest plot). Results: Eight articles were finally included in the selection, involving a total of 1,027 patients. PVP surgery using the new implant-assisted VA technique was superior to PKP surgery in relieving postoperative pain [mean difference $(\mathrm{MD})=-3.77,95 \% \mathrm{CI}:-5.63,-1.92, \mathrm{P}<0.0001]$ and improving the postoperative Oswestry Disability Index (ODI) score ( $M D=-1.59,95 \% \mathrm{CI}:-3.01,-0.16, \mathrm{P}=0.03)$. However, it was not significantly different from PKP surgery in improving postoperative quality of life (MD =-0.27, 95\% CI: $-3.55,3.01, \mathrm{P}=0.87$ ), and the cement extravasation rate was significantly lower than that of PKP surgery [odd ratio $(\mathrm{OR})=0.38,95 \% \mathrm{CI}: 0.19,0.74, \mathrm{P}=0.004]$.

Discussion: The new implant-assisted VA technique can significantly relieve pain, reduce clinical symptoms, improve postoperative quality of life, and significantly reduce the problem of cement extravasation. However, this new technology is still evolving, and more high-quality randomized controlled studies on this topic are needed to provide stronger evidence.

Keywords: Vertebral augmentation (VA); percutaneous vertebroplasty (PVP); percutaneous kyphoplasty (PKP); osteoporotic vertebral compression fracture (OVCF); meta-analysis

Submitted Sep 16, 2021. Accepted for publication Nov 12, 2021.

doi: 10.21037/apm-21-2834

View this article at: https://dx.doi.org/10.21037/apm-21-2834 


\section{Introduction}

Osteoporosis is a metabolic disease that occurs frequently in the elderly ( $>60$ years of age). With aging, the function and activity of osteoblasts of the patients decrease, while osteoclasts increase under the regulation of hormones, which leading to bone formation decreases and bone resorption increases, resulting in bone loss, changes in bone structure, increased bone fragility, and extreme susceptibility to fracture. Osteoporosis vertebral compressed fracture (OVCF) is a common fracture caused by osteoporosis. Thoracic vertebral fractures are manifested as strong pain in the thoracic segment, kyphosis, thoracoabdominal compression, muscle atrophy, loss of self-care ability, and severe cases can cause paralysis and bed rest, or even loss of life (1). The disease can be treated conservatively, which involves analgesia and osteoporosis drugs, resting in bed, and wearing a brace to ensure body balance. However, conservative treatment alone cannot cure the patient's pain condition and the dysfunction caused by the disease (2).

Surgical treatment can restore the physical stability of the lumbar spine, relieve pain, improve function, and enhance the quality of life of patients. At present, the more mature minimally invasive surgeries mainly include percutaneous vertebroplasty (PVP) and percutaneous kyphoplasty (PKP). Both of these procedures enhance the strength and toughness of the vertebral body by injecting fillers (usually bone cement materials, polymethyl acrylate, PMMA) into the vertebral body, which is classified as vertebral augmentation (VA) therapy, and can increase the pain relief rate of patients by more than $90 \%$ (3). Hinde et al. (4) conducted a meta-analysis which included 16 RCT literatures and compared the effects of surgical (PVP or $\mathrm{PKP})$ treatment with non-surgical treatment in more than 2 million patients with OVCF, and showed that the mortality of patients treated with surgery was $22 \%$ lower than that of patients treated with non-surgical treatment after 10 years. Some other studies compared the effects of PVP with PKP, the results of which had suggested that the PKP technique dilates the vertebral body and injects bone cement into the cavity, has lower cement extravasation rate, and reduces spinal cord oppression and nerve root injury caused by leakage, however, with the development of medical science and technology as well as the introduction of some implants, PVP technology corrects the original cement extravasation problem and shows more excellent clinical results (5). Nowadays there was still no such metaanalysis for the new implant-assisted VA techniques. In this study, we searched the literature reporting new PVP techniques in recent years and compared them with PKP by means of meta-analysis to understand the therapeutic effect of the latest VA surgery. We present the following article in accordance with the PRISMA reporting checklist (available at https://dx.doi.org/10.21037/apm-21-2834).

\section{Methods}

\section{Search strategy}

PubMed, Embase, Ovid, and SpringerLink were selected as the main query databases, and data related to VA was searched through other channels such as clinicaltrials.gov, and Google scholar. The mainstream databases were searched using the keyword rapid search: "Vertebral augmentation/ Percutaneous vertebro-plasty/PVP" AND "Osteoporosis/ Vertebral compressed fractures/VCF". If the database had a filter function, the literature publication time was limited from January 2010 to the present, and the literature type was limited to randomized controlled trials (RCTs).

\section{Eligibility indicators}

\section{Study subjects}

Patients diagnosed with osteoporotic vertebral compression fractures (OVCF).

\section{Intervention method}

This study included two groups of patients. The experimental group used the modified version of PVP; i.e., the VA assisted technique, using any minimally invasive implantation mechanical device (most commonly the SpineJack ${ }^{\circledR}$ system, the Kiva ${ }^{\circledR}$ support system, etc.), while the control group underwent PKP.

\section{Outcome indicators}

The primary indicators were the Oswestry Disability Index (ODI), Visual Analogue Scale (VAS), Euro Quality of Life 5-domian Questionnaire (EQ-5D), and Cement extravasation rate $(\mathrm{CE})$, while the secondary indicators included the postoperative subsequent vertebral fractures rate (SVF) and device bolus migration rate (DBM). Considering the need for meta-analysis, the score on all 10 points was extended to the percent scale. If the final VAS score of the 10 -point evaluation (scale range $0-10$ ) is multiplied by 10 , the ODI parameter of 10-point scale (scale range $0-10$ ) is multiplied by 10 , while the score of EQ- 
5D quality of life scale of $0-1$ point is multiplied by 100 , it will be transformed into a scale $0-100$ for meta-analysis. Since the observation time for outcome indicators in the literatures varied, for studies with multiple observation points (such as $5 \mathrm{~d}, 1$ month, 3 months, and 6 months postoperatively), the outcome indicator data at 12 months postoperatively was taken for analysis.

\section{Excluded literature}

(I) Studies with a total sample size less than 10; (II) nonrandomized controlled studies; (III) studies whose data could not be transformed or be used after transformation; and (IV) repeated published literature, or studies with obvious errors in interventions and inaccurate data.

\section{Selection of literature and extraction of data}

Two researchers independently screened the literature and extracted the data. If the data was missing in the literature, we attempted to contact the author for it. The extracted data included the following: author, publication time, study region, intervention method, grouping method, number of cases, follow-up time, and outcome indicators. Two researchers performed cross-examination; disagreement with the data or differences of opinion were settled by inviting a third person for arbitration.

\section{Literature risk of bias assessment and quality assessment}

The risk of literature bias was assessed using the Cochrane randomized controlled trial evaluation tool integrated in the meta-analysis software, Revman 5.4 released by Cochrane Collaboration, to evaluate the following six aspects: randomization method, allocation concealment, blinding method, loss to follow-up and withdrawal, data integrity, and selective reporting of included RCTs. The Jadad scoring criteria were used to evaluate the quality of the included studies, a score of less than 3 was considered lowquality literature, and a score greater than 3 was considered high-quality literature.

\section{Statistical analysis}

(I) Tools: Revman 5.4 was used as the analysis tool for this study. (II) Effect size: for the continuous indicators of VAS score, ODI score, and EQ-5D, the mean difference (MD) and $95 \%$ Confidential Interval (CI) were considered to indicate the effect size; for the binary variables of cement extravasation rate and subsequent fracture; the odds ratio $(\mathrm{OR})$ and $95 \% \mathrm{CI}$ were considered to indicate the effect size. $\mathrm{P}<0.05$ was considered to indicate a statistically significant difference in effect size, and a forest plot was used to present the effect size; studies reporting these indicators would be included for synthesis. (III) Effect model: a random-effects model was used if there was heterogeneity between the articles; otherwise, a fixed effect was used. (IV) Heterogeneity: $\mathrm{I}^{2}$ analysis and $\mathrm{Q}$ test were used for analysis; $\mathrm{I}^{2}>50 \%$ or $\mathrm{P}<0.1$ indicated statistically significant heterogeneity. (V) Heterogeneity and sensitivity survey: the piecemeal exclusion method was used for analysis. (VI) Publication bias report: funnel plots were used to report publication bias.

\section{Results}

\section{Literature screening process and results}

The literature screening flowchart is shown in Figure 1. In total, 418 articles were initially retrieved, including 355 studies retrieved from mainstream databases and 63 literatures from other sources. Finally, eight studies were included and selected, with a total of 1,027 patients, as shown in Figure 1.

\section{Basic characteristics of literatures and intervention methods}

The basic characteristics and intervention methods of the included articles are shown in Table 1.

\section{Risk analysis of literature bias}

Among the eight RCTs included in this study, all mentioned the randomization method, no allocation concealment was mentioned in the literatures $(7,9,12)$, no blinding methods were mentioned in the literatures (9), which meant that the final results possibly had selection and implementation bias. The dropout cases in 8 literatures were described in detail, so there was no detection deviation and incompleteness. There were no selective reports and other biases in the literature, as shown in Figures 2,3.

\section{Meta-analysis results}

Effect of PVP VA on relieving postoperative pain All included studies reported postoperative pain. The heterogeneity $\left(\mathrm{I}^{2}=14 \%, \mathrm{P}=0.32\right)$ was not statistically 
Identification of studies via databases and registers
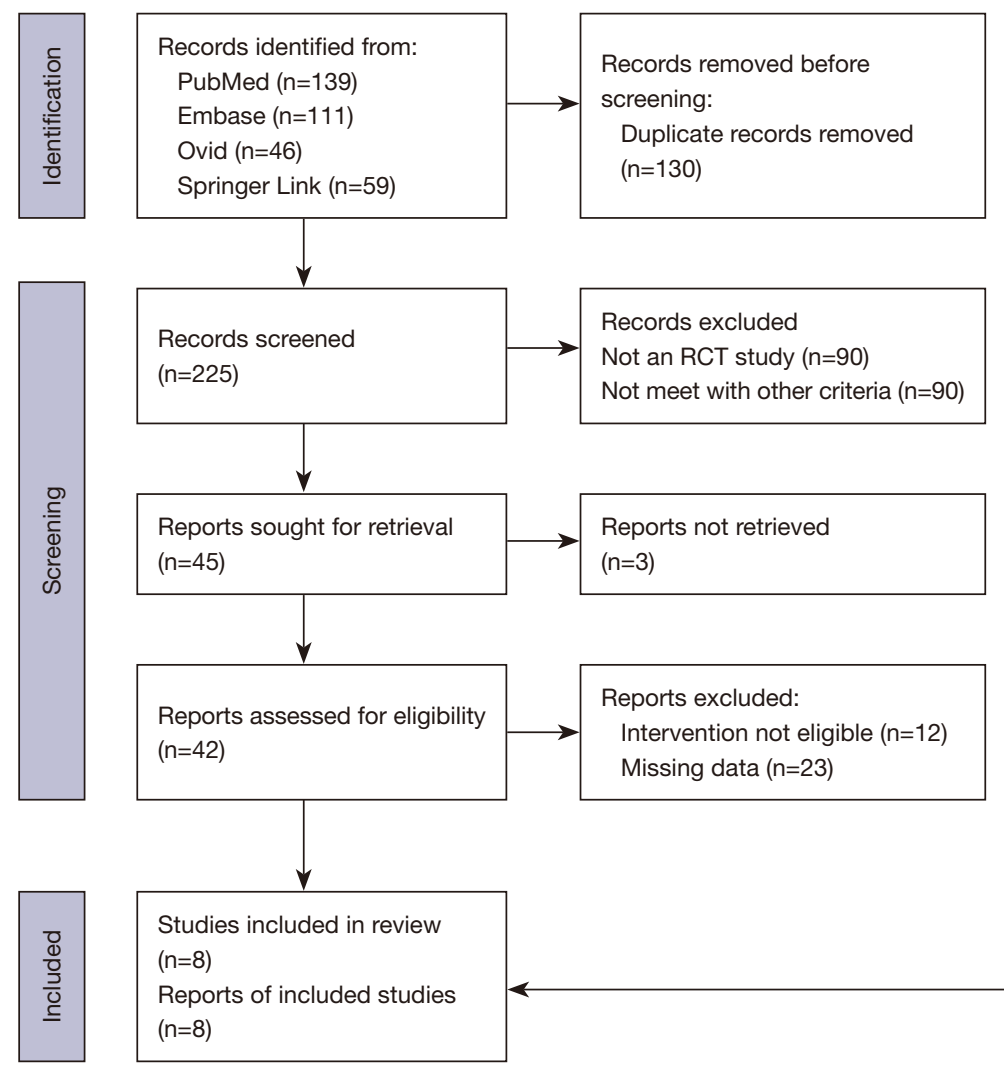

$(n=225)$
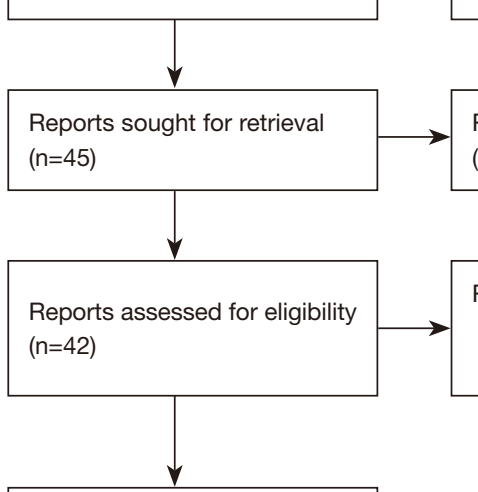

Studies included in review

$(n=8)$

Reports of included studies

$(n=8)$
Identification of studies via other methods

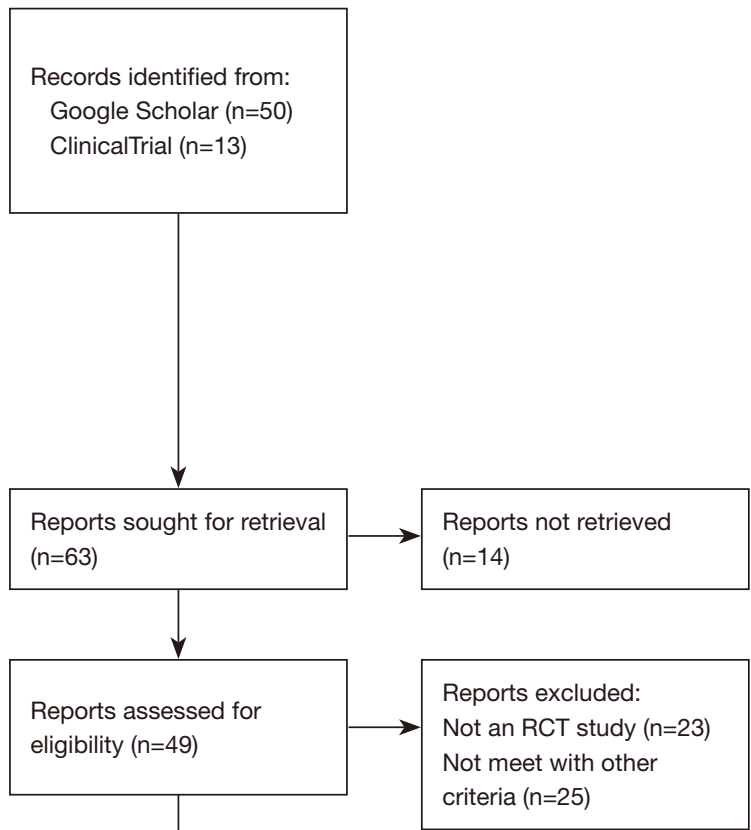

Figure 1 Literature selection flow diagram.

significant; thus, the fixed effect model was used to obtain a combined effect size of $\mathrm{MD}=-3.77,95 \% \mathrm{CI}:-5.63$, -1.92 . The combined effect size showed that the relief of postoperative pain after PVP VA in the experimental group was superior to that of the control group $(Z=3.98$, $\mathrm{P}<0.0001)$. The number of cases in the meta-analysis excluded all cases of dropout and loss. Therefore, there was no data incompleteness (Figure 4).

\section{Effect of PVP VA on improving the postoperative ODI functional index in patients}

Only one study (13) did not report the postoperative ODI index improvement, while the other seven articles reported that the heterogeneity of literature $\left(\mathrm{I}^{2}=44 \%, \mathrm{P}=0.10\right)$ was not statistically significant. Using the fixed effect model, a pooled effect size (MD $=-1.59$, 95\% CI: $-3.01,-0.16)$ was obtained. The pooled effect size showed that PVP VA in the experimental group was superior to the control group in improving the postoperative ODI index $(\mathrm{Z}=2.18, \mathrm{P}=0.03)$
(Figure 5).

Effect of PVP VA on the improvement of postoperative quality of life

A total of five articles $(6,7,9-11)$ reported the postoperative quality of life, but one study (10) used SF-36 scale statistics and was thus excluded. The remaining four RCTs had no statistical heterogeneity $\left(\mathrm{I}^{2}=0.0 \%, \mathrm{P}=0.54\right)$; using the fixed effect model, the combined statistics were obtained (MD $=-0.27,95 \% \mathrm{CI}:-3.55,3.01)$, and the difference was not statistically significant $(\mathrm{Z}=0.16, \mathrm{P}=0.87)$. Therefore, the improvement in postoperative quality of life with PVP VA was not significantly different from the control group, as shown in Figure 6.

\section{Evaluation of cement extravasation in PVP VA}

A total of four articles (6,8-10) reported the cement extravasation rate, and there was no heterogeneity in the studies $\left(\mathrm{I}^{2}=0.0 \%, \mathrm{P}=0.41\right)$. Using the fixed effect model, the 
Table 1 Basic characteristics and intervention methods of included literatures

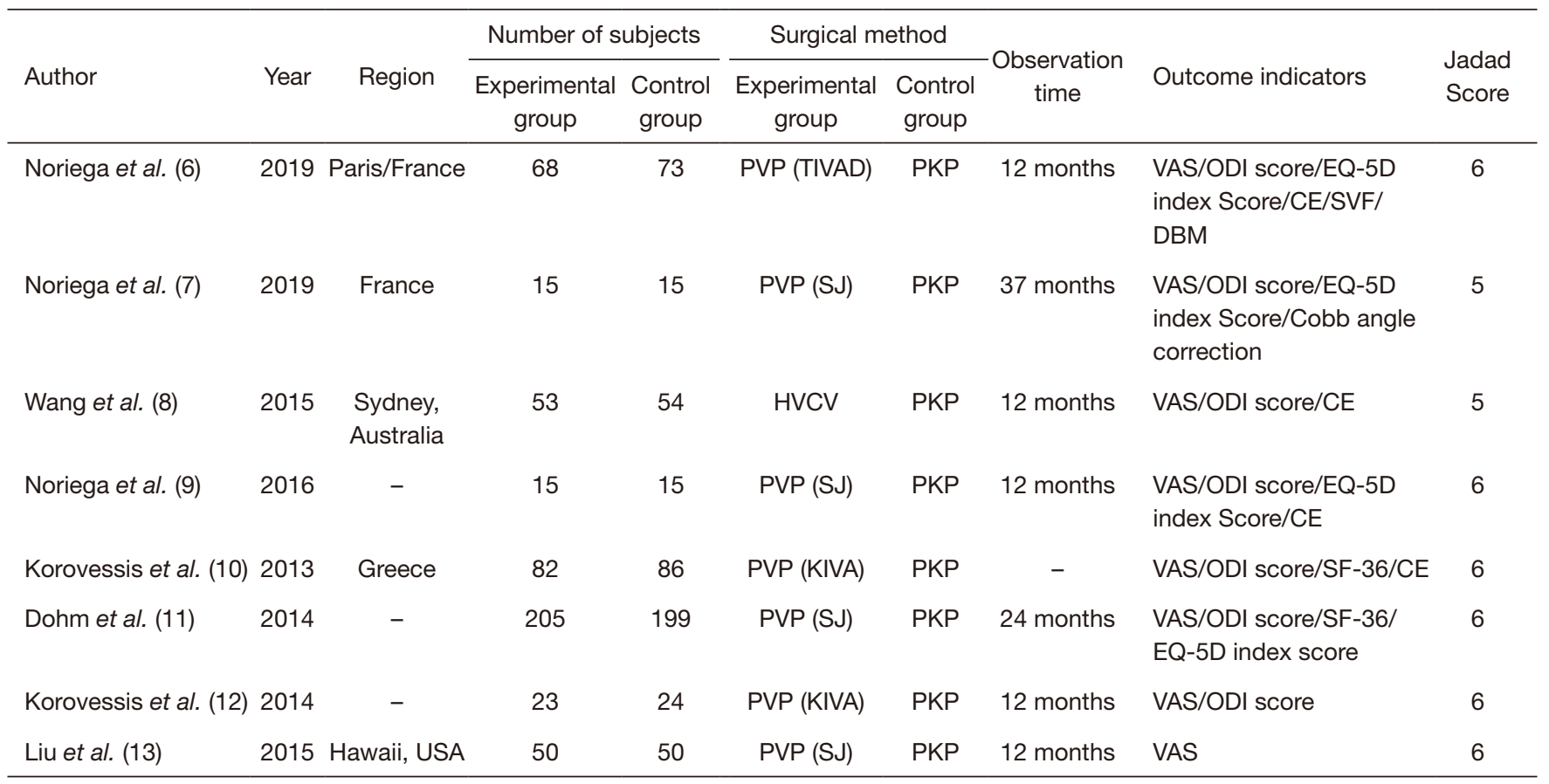

VA, vertebral augmentation; TIVAD, titanium implantable vertebral augmentation device; PKP, balloon kyphoplasty; VAS, visual analogue score; ODI, Oswestry Disability Index; EQ-5D, Euro Quality of Life 5-domian Questionnaire; CE, cement extravasation; SVF, subsequent vertebral fractures; DBM, device bolus migration; SJ, SpineJack ${ }^{\circledR}$; HVCV, high viscosity cement vertebroplasty; KIVA, a novel vertebral augmentation technique.

pooled statistic was OR $=0.38$ (95\% CI: $0.19,0.74$ ), and the difference was statistically significant $(\mathrm{Z}=2.87, \mathrm{P}=0.004)$. Thus, the cement extravasation rate in PVP VA was significantly lower than that in the control group (Figure 7).

\section{Heterogeneity investigation and sensitivity analysis}

The four analyses in this study all showed that there was no heterogeneity among the included studies, so no indepth heterogeneity investigation performed. We used the random effect model for extra analysis and results were almost the same with the fixed effect model, which should the results were stable.

\section{Publication bias analysis}

The funnel plot of the pain VAS index synthesis showed an even distribution of the studies, which meant that there was little publication bias among the studies, as Figure 8.

\section{Discussion}

OVCF is a common complication in patients with osteoporosis, compared with the conservative treatment of only using drugs and braces, surgical treatment can reduce pain and improve the ability of movement, if the patients do not undergo surgical treatment in time, the vertebral body may continue to collapse, the activity ability of the patient will be weakened, the pain can not be relieved, which result in a low quality of life (14). VA is the main surgical method for OVCFs, and is characterized by reduced trauma, less bleeding, rapid postoperative recovery, and high safety (15). PVP and PKP are the two most common types of VA. The PVP technique injects bone cement into the vertebral body under high pressure and is easy to operate. Owing to the fact that there is no need to place a dilator, it is not necessary to repeatedly puncture to establish a dilator placement channel, and the puncturerelated complications are minimal (16). However, PVP has no fixed space to contain bone cement, and some studies have reported that its cement extravasation rate can reach $11-76 \%$. However, the strict surgical procedure, coupled with the extensive experience of doctors, can minimize the cement extravasation rate, and therefore, PVP is still a cost-effective surgical method (17). PKP creates a cavity by placing a dilator (usually a balloon) in the patient's 
vertebral body and injecting bone cement into the cavity, and the rate of cement extravasation is greatly reduced compared with PVP, with high safety (18). However, with the development of implant-assisted technology, the technical function of PVP has been improved and is showing excellent efficacy (5).

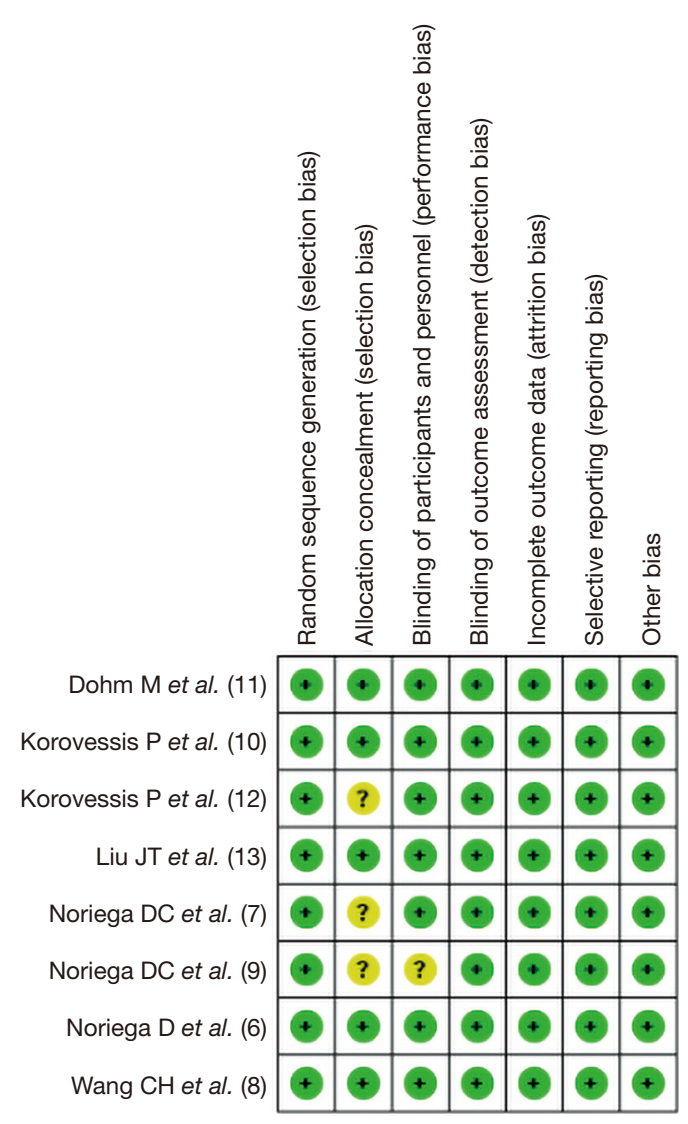

Figure 2 Brief introduction of the literature bias risk.
In this meta-analysis, a total of eight articles were selected from literature databases. Among the included studies, the intervention measures in the experimental group were PVP surgery using implant-assisted technique, while those in the control group were PKP. The study results showed that the experimental group was superior to the control group in the relief of pain and improvement of the functional index. There was no significant difference in the improvement of quality of life between the two procedures. However, the bone cement extravasation rate in the experimental group was significantly lower than that in the control group. A study by Filippiadis et al. (19) had pointed out that although PKP surgery can rapidly correct kyphotic deformity and relieve pain, it also exhibits disadvantages in application, such as the problem of cement extravasation, damage to bone structure during balloon placement and withdrawal, and the problem of vertebral body re-collapse after balloon withdrawal, which can aggravate the kyphotic deformity of patients and affect the quality of life of patients. Spinal implantation aids are the product of the development of new technologies in recent years. The principle is to place the device through the pedicle or posterolateral vertebral body, distract the vertebral body, correct kyphotic deformity, form an intravertebral cavity, and then inject bone cement for reinforcement, so as to achieve the effect of classical PVP surgery, while preventing bone cement extravasation and reducing recurrent fractures, thereby achieving a superior effect (5).

In this study, since all eight included studies reported the index data after a 12 -month observation period, while some studies missed the early and long-term postoperative index, this study only reflected the effect of surgery in the two groups at the mid-term postoperative period (12 months). Moreover, there are various types of implant-

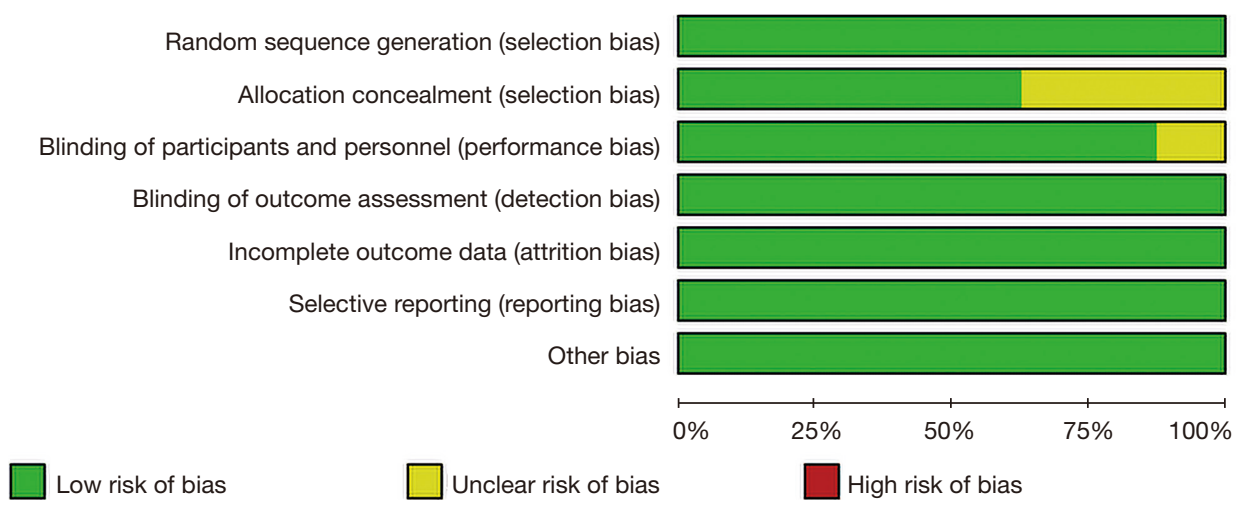

Figure 3 Literature bias risk assessment table. 


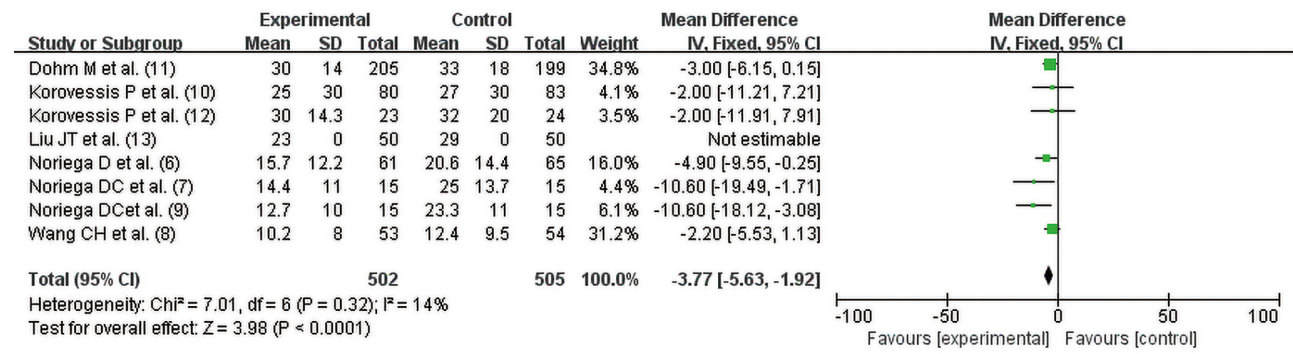

Figure 4 Combined effect plot of PVP VA on pain relief. PVP, percutaneous vertebroplasty; VA, vertebral augmentation.

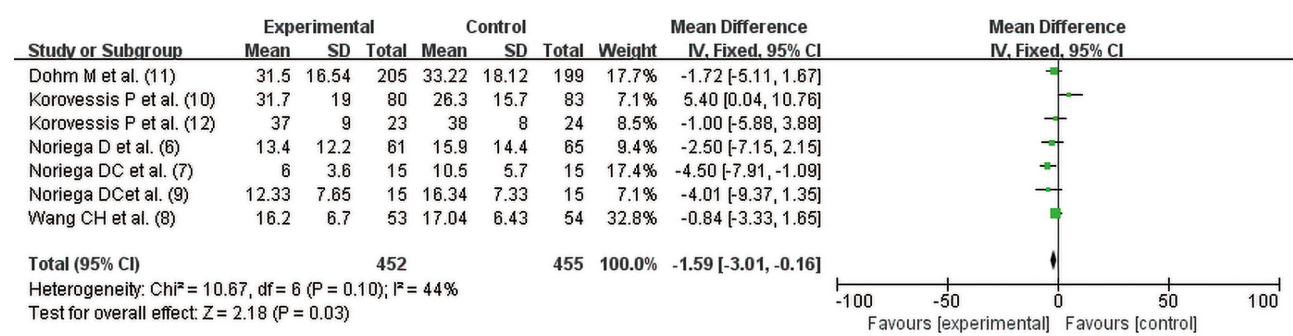

Figure 5 Combined effect plot of PVP VA for improving ODI. PVP, percutaneous vertebroplasty; VA, vertebral augmentation; ODI, Oswestry Disability Index.

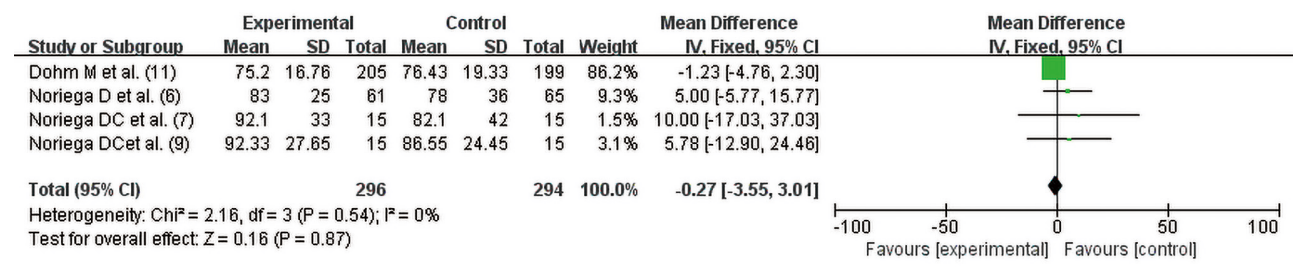

Figure 6 Combined effect diagram of PVP VA in improving the quality of life of patients. PVP, percutaneous vertebroplasty; VA, vertebral augmentation.

\begin{tabular}{|c|c|c|c|c|c|c|c|c|c|c|}
\hline \multirow{2}{*}{ Studv or Subgroup } & \multicolumn{2}{|c|}{ Experimental } & \multicolumn{2}{|c|}{ Control } & \multirow{2}{*}{\multicolumn{2}{|c|}{$\begin{array}{cc} & \text { Odds Ratio } \\
\text { Weight } & \text { M-H, Fixed, } 95 \% \mathrm{Cl} \\
\end{array}$}} & \multirow{2}{*}{\multicolumn{4}{|c|}{$\begin{array}{c}\text { Odds Ratio } \\
\text { M-H, Fixed, 95\% Cl }\end{array}$}} \\
\hline & & Total & Events & Total & & & & & & \\
\hline Korovessis $\mathrm{P}$ et al. (10) & 4 & 82 & 12 & 86 & $37.0 \%$ & $0.32[0.10,1.02]$ & & & & \\
\hline Noriega D et al. (6) & 5 & 75 & 12 & 80 & & Not estimable & & & & \\
\hline Noriega DCet al. (9) & 1 & 15 & 0 & 15 & $1.5 \%$ & $3.21[0.12,85.20]$ & & & & \\
\hline Wang $\mathrm{CH}$ et al. (8) & 9 & 68 & 22 & 72 & $61.5 \%$ & $0.35[0.15,0.82]$ & & & & \\
\hline Total $(95 \% \mathrm{Cl})$ & & 165 & & 173 & $100.0 \%$ & $0.38[0.19,0.74]$ & & & & \\
\hline Total events & 14 & & 34 & & & & & & & \\
\hline $\begin{array}{l}\text { Heterogeneity: } \mathrm{Chi}^{2}=1.7 \\
\text { Test for overall effect: } Z=\end{array}$ & $\begin{array}{l}\text { df }=2(P \\
2.87(P=\end{array}$ & $\begin{array}{l}=0.41) ; \\
.004)\end{array}$ & $F^{2}=0 \%$ & & & & 0.01 & $\begin{array}{l}0.1 \\
\text { s [experimental] }\end{array}$ & $1 \frac{10}{\text { Favours [contro }}$ & 100 \\
\hline
\end{tabular}

Figure 7 Combined effect plot of PVP VA for reducing cement extravasation rate. PVP, percutaneous vertebroplasty; VA, vertebral augmentation.

assisted technologies. In addition to the TIVAD device, SpineJack device, and KIVA device included in this study, other more advanced technologies have been utilized, such as the Osseofix system (US) (20) and vertebral body stenting (VBS) system (Switzerland) (21). However, there were no studies that could be included in this meta- 


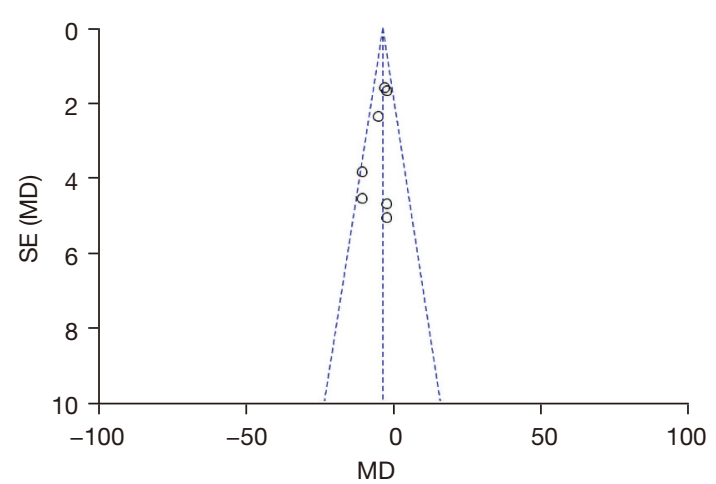

Figure 8 Plot of publication bias.

analysis.

\section{Conclusions}

In summary, this meta-analysis included eight studies on VA in the treatment of OVCF, with a low risk of bias and conclusive evidence, showing that the new technique assisted by VA implant can significantly relieve patients' pain, reduce clinical symptoms, improve the postoperative quality of life, and significantly reduce the problem of bone cement extravasation. However, this new technology is still evolving, and more high-quality randomized controlled studies on this topic are needed to provide stronger evidence.

\section{Acknowledgments}

Funding: This work was supported by the first batch of major scientific research projects of Sichuan Provincial Department of Science and Technology in 2020 (2020YFS0416).

\section{Footnote}

Reporting Checklist: The authors have completed the PRISMA reporting checklist. Available at https://dx.doi. org/10.21037/apm-21-2834

Conflicts of Interest: All authors have completed the ICMJE uniform disclosure form (available at https://dx.doi. org/10.21037/apm-21-2834). The authors have no conflicts of interest to declare.

Ethical Statement: The authors are accountable for all aspects of the work in ensuring that questions related to the accuracy or integrity of any part of the work are appropriately investigated and resolved.

Open Access Statement: This is an Open Access article distributed in accordance with the Creative Commons Attribution-NonCommercial-NoDerivs 4.0 International License (CC BY-NC-ND 4.0), which permits the noncommercial replication and distribution of the article with the strict proviso that no changes or edits are made and the original work is properly cited (including links to both the formal publication through the relevant DOI and the license). See: https://creativecommons.org/ licenses/by-nc-nd/4.0/.

\section{References}

1. Imai K. Vertebral fracture risk and alendronate effects on osteoporosis assessed by a computed tomography-based nonlinear finite element method. J Bone Miner Metab 2011;29:645-51.

2. Zhao WT, Qin DP, Zhang XG, et al. Biomechanical effects of different vertebral heights after augmentation of osteoporotic vertebral compression fracture: a threedimensional finite element analysis. J Orthop Surg Res 2018;13:32.

3. Zhou T, Lin H, Wang H, et al. Comparative study on the biomechanics between improved PVP and traditional PKP in the treatment of vertebral peripheral wall damage-type OVCF. Exp Ther Med 2017;14:575-80.

4. Hinde K, Maingard J, Hirsch JA, et al. Mortality Outcomes of Vertebral Augmentation (Vertebroplasty and/or Balloon Kyphoplasty) for Osteoporotic Vertebral Compression Fractures: A Systematic Review and MetaAnalysis. Radiology 2020;295:96-103.

5. Premat K, Vande Perre S, Cormier É, et al. Vertebral augmentation with the SpineJack ${ }^{\circledR}$ in chronic vertebral compression fractures with major kyphosis. Eur Radiol 2018;28:4985-91.

6. Noriega D, Marcia S, Theumann N, et al. A prospective, international, randomized, noninferiority study comparing an implantable titanium vertebral augmentation device versus balloon kyphoplasty in the reduction of vertebral compression fractures (SAKOS study). Spine J 2019;19:1782-95.

7. Noriega DC, Rodríguez-Monsalve F, Ramajo R, et al. Long-term safety and clinical performance of kyphoplasty and SpineJack ${ }^{\circledR}$ procedures in the treatment 
of osteoporotic vertebral compression fractures: a pilot, monocentric, investigator-initiated study. Osteoporos Int 2019;30:637-45.

8. Wang CH, Ma JZ, Zhang CC, et al. Comparison of highviscosity cement vertebroplasty and balloon kyphoplasty for the treatment of osteoporotic vertebral compression fractures. Pain Physician 2015;18:E187-94.

9. Noriega DC, Ramajo RH, Lite IS, et al. Safety and clinical performance of kyphoplasty and SpineJack(®) procedures in the treatment of osteoporotic vertebral compression fractures: a pilot, monocentric, investigator-initiated study. Osteoporos Int 2016;27:2047-55.

10. Korovessis P, Vardakastanis K, Repantis T, et al. Balloon kyphoplasty versus KIVA vertebral augmentation-comparison of 2 techniques for osteoporotic vertebral body fractures: a prospective randomized study. Spine (Phila Pa 1976) 2013;38:292-9.

11. Dohm M, Black CM, Dacre A, et al. A randomized trial comparing balloon kyphoplasty and vertebroplasty for vertebral compression fractures due to osteoporosis. AJNR Am J Neuroradiol 2014;35:2227-36.

12. Korovessis P, Vardakastanis K, Vitsas V, et al. Is Kiva implant advantageous to balloon kyphoplasty in treating osteolytic metastasis to the spine? Comparison of 2 percutaneous minimal invasive spine techniques: a prospective randomized controlled short-term study. Spine (Phila Pa 1976) 2014;39:E231-9.

13. Liu JT, Li CS, Chang CS, et al. Long-term follow-up study of osteoporotic vertebral compression fracture treated using balloon kyphoplasty and vertebroplasty. J Neurosurg Spine 2015;23:94-8.

14. Pourtaheri S, Luo W, Cui C, et al. Vertebral Augmentation is Superior to Nonoperative Care at Reducing Lower Back Pain for Symptomatic

Cite this article as: Lin S, Cheng M, Liang W, Yuan X. Efficacy of vertebral augmentation in the treatment of osteoporotic vertebral compression fractures: a systematic review and meta-analysis. Ann Palliat Med 2021;10(11):1176711775. doi: 10.21037/apm-21-2834
Osteoporotic Compression Fractures: A Meta-Analysis. Clin Spine Surg 2018;31:339-44.

15. Sun Y, Ma H, Yang F, et al. Clinical Efficacy and Safety of Zoledronic Acid Combined with PVP/PKP in the Treatment of Osteoporotic Vertebral Compression Fracture: A Systematic Review and Meta-Analysis of Randomized Controlled Trials. Biomed Res Int 2021;2021:6650358.

16. Chang X, Lv YF, Chen B, et al. Vertebroplasty versus kyphoplasty in osteoporotic vertebral compression fracture: a meta-analysis of prospective comparative studies. Int Orthop 2015;39:491-500.

17. Buchbinder R, Johnston RV, Rischin KJ, et al. Percutaneous vertebroplasty for osteoporotic vertebral compression fracture. Cochrane Database Syst Rev 2018;4:CD006349.

18. Diel P, Röder C, Perler G, et al. Radiographic and safety details of vertebral body stenting: results from a multicenter chart review. BMC Musculoskelet Disord 2013;14:233.

19. Filippiadis DK, Marcia S, Masala S, et al. Percutaneous Vertebroplasty and Kyphoplasty: Current Status, New Developments and Old Controversies. Cardiovasc Intervent Radiol 2017;40:1815-23.

20. Long Y, Yi W, Yang D. Advances in Vertebral Augmentation Systems for Osteoporotic Vertebral Compression Fractures. Pain Res Manag 2020;2020:3947368.

21. Li C, Zhang HB, Zhang H, et al. Severe pathological fractures caused by vertebral hemangiomas with posterior decompression, bone cement augmentation and internal fixation. Orthop Traumatol Surg Res 2016;102:489-94.

(English Language Editor: A. Kassem) 